\title{
Effect of immune suppression on metastasis in a patient with hepatocellular carcinoma metastasized to the colon and stomach: A case report
}

\author{
DONGDE WU ${ }^{1}$, SHAOZHONG WEI ${ }^{2}$, BAOZHEN LIU ${ }^{3}$, XINGHONG WU $^{4}$, YAOJUN FENG $^{4}$, \\ CHENGGANG LUO ${ }^{1}$, YIQING JU ${ }^{5}$ and JIANJUN LIANG ${ }^{5}$ \\ ${ }^{1}$ Department of Hepatobiliary and Pancreatic Surgery; ${ }^{2}$ Department of Urology Surgery, \\ Cancer Hospital of Wuhan University (Hubei Cancer Hospital), Wuhan, Hubei 430079; \\ ${ }^{3}$ School Hospital of Wuhan Textile University, Wuhan, Hubei 430073; ${ }^{4}$ Department of Breast Cancer Surgery, \\ Cancer Hospital of Wuhan University (Hubei Cancer Hospital), Wuhan, Hubei 430079; \\ ${ }^{5}$ Department of General Surgery, First Hospital of Wuxue County, Huanggang, Hubei 435400, P.R. China
}

Received December 3, 2014; Accepted January 21, 2016

DOI: $10.3892 / \mathrm{etm} .2016 .3108$

\begin{abstract}
Hepatocellular carcinoma (HCC) is a highly malignant cancer, which can invade the portal vein and cause liver/long bone metastasis, although digestive tract metastatic tumor from the liver is very rare. This case report describes an unusual case of HCC (clear cell type), determined by pathology of the original liver tumor resected on March 16th, 2004. The patient returned to our hospital in February and July 2009 complaining of 'black stool' in the first instance, and 'anemia' on the second occasion. Colonoscopy and gastroscopy indicated colon cancer and stomach cancer, respectively. The right half colon and distal stomach were resected, and pathological inspection revealed liver cancer metastasis. The patient succumbed to respiratory failure due to liver cancer lung metastasis on the May 23rd, 2013. Tests for $\mathrm{CD}^{+}$and $\mathrm{CD}^{+} \mathrm{T}$ cells and the $\mathrm{CD} 4^{+} / \mathrm{CD}^{+}$ratio, in addition to the expression of Fas, Fas ligand (FasL), indicated an evident difference in patient immunity during the tumor metastasis period. The disease progression in this patient suggested that immune surveillance may have been involved in the metastases. Furthermore, this case shows that clinicians should be alert to the possibility of metastases in uncommon sites that may be misdiagnosed as primary tumors. Surgical resection remains a valuable treatment for isolated digestive tract metastasis from liver cancer.
\end{abstract}

Correspondence to: Dr Shaozhong Wei, Department of Urology Surgery, Cancer Hospital of Wuhan University (Hubei Cancer Hospital), 116 Zhuodaoquan South Road, Hongshan, Wuhan, Hubei 430079, P.R. China

E-mail:971070316@qq.com

Key words: hepatocellular carcinoma, immunosuppression, colon metastasis, stomach metastasis, prognosis

\section{Introduction}

Due to the prevalence of malignancy, the prognosis for hepatocellular carcinoma (HCC) is poor. The major factors affecting prognosis are recurrence and metastasis. Patients with HCC frequently show intrahepatic metastasis and extrahepatic metastasis to lung and bone $(1,2)$. However, more recent reports have shown that HCC can spread to unusual extrahepatic sites including the gallbladder, distal pancreas, chest wall and skin (3-6). Terada and Maruo (7) reported autopsy findings which indicated that extrahepatic metastases were present in $68 \%$ of HCC cases, with $59 \%$ of metastatic sites found in the lungs. The present study reports an HCC patient that was followed up for $>9$ years following a primary liver tumor resection and in which metachronous colon and stomach metastases were observed. To the best of our knowledge, HCC metastases to the colon and stomach in the same patient have not previously been reported.

There may be an association between tumor progression and immune status, such that the failure of the immune system to identify tumorous cells results in the spread of cancer cells to other organs (8). It has been shown that Fas/FasL-mediated apoptosis and maintenance of immunological self-tolerance (9). The Fas ligand (Fas) is a cell surface tumor necrosis factor-family member receptor, associated with the cellular apoptosis signal transmission system (9). Fas, also known as CD95, is a receptor molecule composed of 325 amino acids (9). Fas exists on the surface of numerous types of cell, while FasL primarily appears on activated T cells and natural killer cells (9). Recent studies have shown that the cellular ratio of immune tolerance in tissues and blood is a powerful marker for tumor aggressiveness and disease dissemination $(10,11)$. During the follow-up of the present case, we aimed to investigate whether the Fas/FasL signal transmission system influenced metastasis, whether cancer metastasis was associated with immune status and whether rare site metastases were associated with severe immunosuppression. Thus, the present case report describes 
the immune status and Fas/FasL, $\mathrm{CD} 4^{+} / \mathrm{CD}^{+}{ }^{+} \mathrm{T}$ cell expression levels at different time points during disease progression in a patient with HCC, and indicates the involvement of the human immune system in the control of tumor metastasis to uncommon sites.

\section{Case report}

Clinical data. A 54-year-old male patient presented with the complaint of a 'right upside abdominal pain for half a month.' A computed tomography (CT) scan (SOMATOM Definition AS; Siemens AG, Munich, Germany) and ultrasound detected a quasi-circular tumor in liver segments IV and $\mathrm{V}(3 \times 3 \times 4 \mathrm{~cm})$. The patient was subsequently admitted to the Hubei Cancer Hospital (Wuhan, China) in February 2004. The liver tumor was resected on March 16th, 2004, and was determined by a pathological examination to be HCC, according to World Health Organization Classification criteria (12). The patient returned to the Hubei Cancer Hospital 5 years later with a gastrointestinal hemorrhage, and a colonoscopy showed an ileocecal junction tumor. A right-half colon cancer radical resection was performed on March 4th, 2009. Postoperative pathological diagnosis indicated an ascending colon infiltrating carcinoma. Metastatic HCC was finally diagnosed when considered in conjunction with the medical history and pathological results. The patient returned a third time with serious anemia, and a gastric body tumor was detected using a gastroscope. A radical extremital partial gastrectomy was performed on July 14th, 2009. Postoperative pathological diagnosis was gastric metastatic HCC (Fig. 1). The patient succumbed to respiratory failure due to liver cancer lung metastasis on May 23rd, 2013. Written informed consent was obtained from the patient's family. The present study was approved by the Ethical Committee of Hubei Cancer Hospital.

Detection of immunological markers. In order to elucidate the HCC metastasis pathology of in the present patient at $>5$ years following the initial tumor resection, immune system alterations in the plasma extracted prior to and following resections of the primary liver cancer and before colon, gastric and lung metastases, were examined. Using flow cytometric analysis to quantify T-lymphocyte subsets, alterations in the populations of $\mathrm{CD}^{+}$and $\mathrm{CD} 8^{+} \mathrm{T}$-cells and the $\mathrm{CD} 4^{+} / \mathrm{CD} 8^{+}$ratio at each time point were observed (Fig. 2). In addition, the expression of Fas, FasL, CD4 ${ }^{+}$and $\mathrm{CD}^{+}$was immunohistochemically evaluated in tumor tissue obtained from the primary liver cancer, ascending colon metastasis and gastric metastasis using a Dako EnVision ${ }^{\mathrm{TM}}$ two-step method (Dako Denmark A/S, Glostrup, Denmark). Fas expression was negative, while FasL expression was positive on the surface of the cancer cells in the primary liver cancer, ascending colon metastasis and gastric metastatic tumor samples (Figs. 3 and 4). Furthermore, $\mathrm{CD}^{+} \mathrm{T}$ cell expression was negative and $\mathrm{CD}^{+} \mathrm{T}$ cell expression was positive in primary liver cancer, ascending colon and gastric metastatic tumor samples (Figs. 5 and 6).

Flow cytometry was conducted using a BD FACSCalibur ${ }^{\mathrm{TM}}$ Flow Cytometer (342975; BD Biosciences, Franklin Lakes, NJ, USA). A Fas, FasL, $\mathrm{CD} 4^{+}$and $\mathrm{CD} 8^{+}$mouse anti-human monoclonal antibody kit was obtained from Dako Denmark A/S.
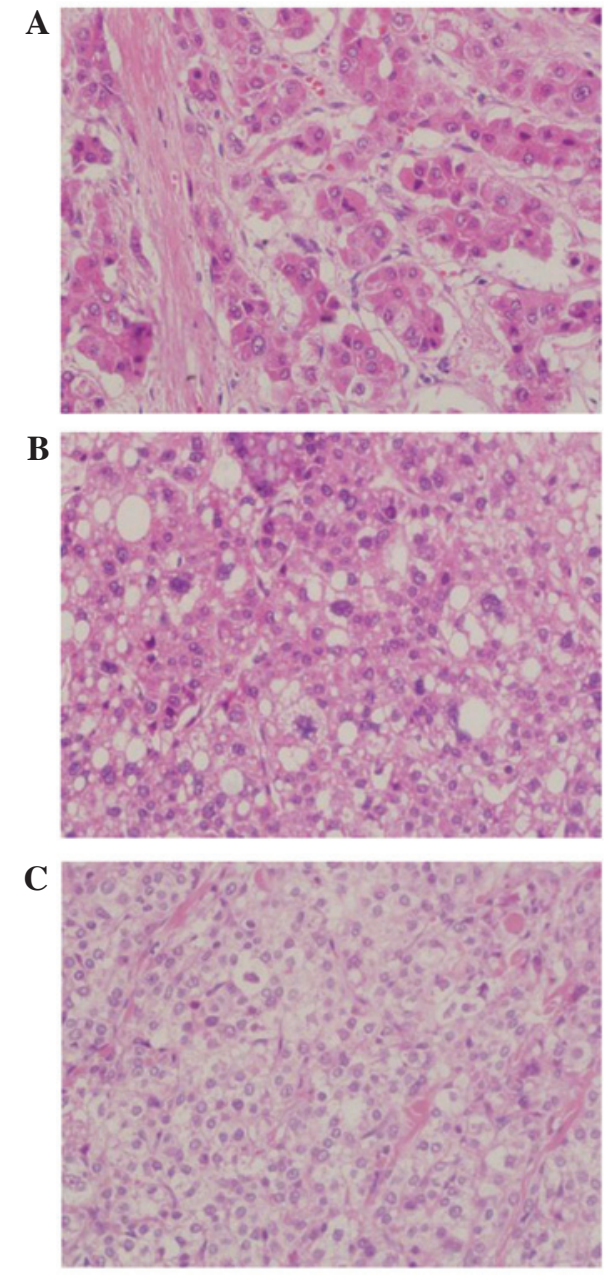

Figure 1. Tumor biopsy specimen of the primary liver cancer and metastases (stain, hematoxylin-eosin; magnification, x40). The cells were polygonal and arranged as closely packed nests. (A) Primary hepatocellular carcinoma (HCC). The well-differentiated HCC had a thin trabecular pattern, and the bland tumor cells had an abundant eosinophilic cytoplasm. (B) Ascending colon metastatic tumor of the same patient. The tumor was composed of atypical cells with an increased nucleus-to-cytoplasm ratio in a thick trabecular or solid pattern. (C) Gastric metastatic tumor. The tumor appeared to grow in a solid pattern.

Follow-up. The patient was monitored closely for 9 years and 2 months from the initial hospital admission to mortality. For the initial 2 years following primary tumor resection, the patient underwent abdominal CT scans, chest radiography and $\alpha$-fetoprotein testing every 3 months, which altered to every 6 months until 5 years post-operatively.

\section{Discussion}

Metachronous cancer metastasis is a common challenge for clinicians, particularly rare site metastatic tumors which are easily missed, thus resulting in misdiagnosis or therapy delay, indicating the requirement for increased study in this area (13).

The digestive tract is not a frequent target of tumor metastasis (14). Gastric metastatic tumors derive predominantly from malignant melanoma, breast cancer, esophageal cancer and kidney cancer, with an incidence of $<0.7 \%$ of all gastric malignant tumors (15). Oda et al (16) reported the 


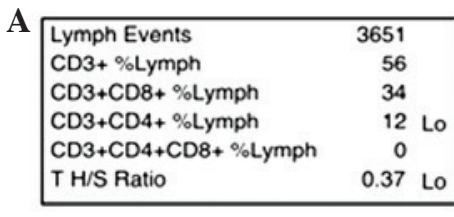

Before primary liver tumor resection

\begin{tabular}{|lr|}
\hline Lymph Events & 4892 \\
CD3+\%Lymph & 65 \\
CD3+CD8+\%Lymph & 20 \\
CD3+CD4+\%Lymph & 45 \\
CD3+CD4+CD8+\%Lymph & 1 \\
T H/S Ratio & 2.27 \\
\hline
\end{tabular}

After primary liver tumor resection

\begin{tabular}{|lr|}
\hline Lymph Events & 2267 \\
CD3+\%Lymph & 84 \\
CD3+CD8+\%Lymph & $50 \quad \mathrm{Hi}$ \\
CD3+CD4+\%Lymph & 25 Lo \\
CD3+CD4+CD8+\%Lymph & 0 \\
T HSS Ratio & 0.49 Lo \\
\hline
\end{tabular}

Before colon metastasis resection

\begin{tabular}{|lr|}
\hline Lymph Events & 2551 \\
CD3+\%Lymph & 83 \\
CD3+CD8+\%Lymph & $55 \mathrm{Hi}$ \\
CD $3+C D 4+\%$ Lymph & $27 \mathrm{LO}$ \\
CD3+CD4+CD8+\%Lymph & 0 \\
T H/S Ratio & 0.49 Lo \\
\hline
\end{tabular}

Before stomach metastasis resection

\begin{tabular}{|lr|}
\hline Lymph Events & 2516 \\
CD3+\%Lymph & 77 \\
CD3+CD8+\%Lymph & $50 \mathrm{Hi}$ \\
CD3+CD4+\%Lymph & $19 \mathrm{LO}$ \\
CD3+CD4+CD8+\%Lymph & 0 \\
T H/S Ratio & $0.38 \mathrm{LO}$ \\
\hline
\end{tabular}

Before found lung metastasis
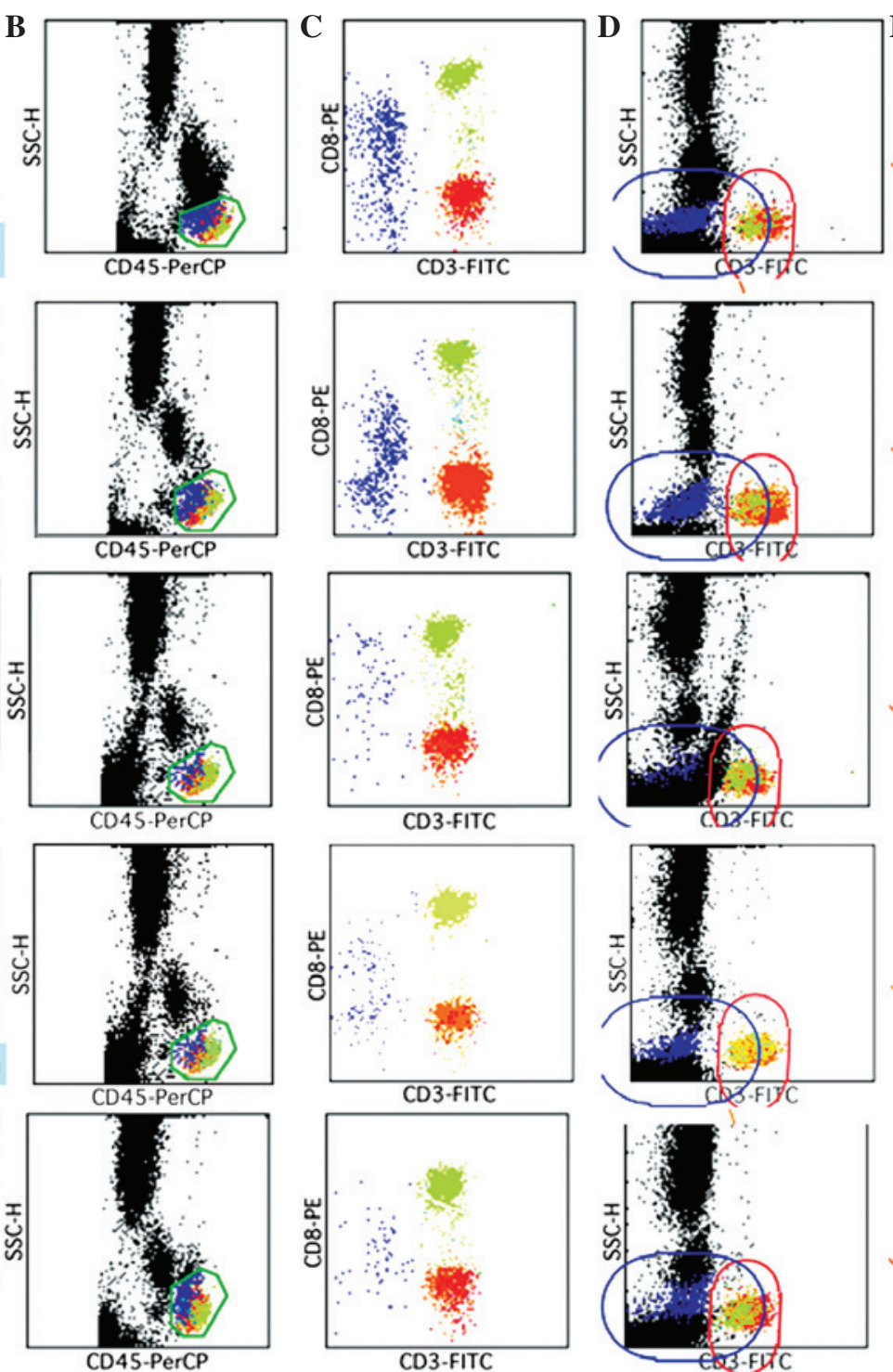
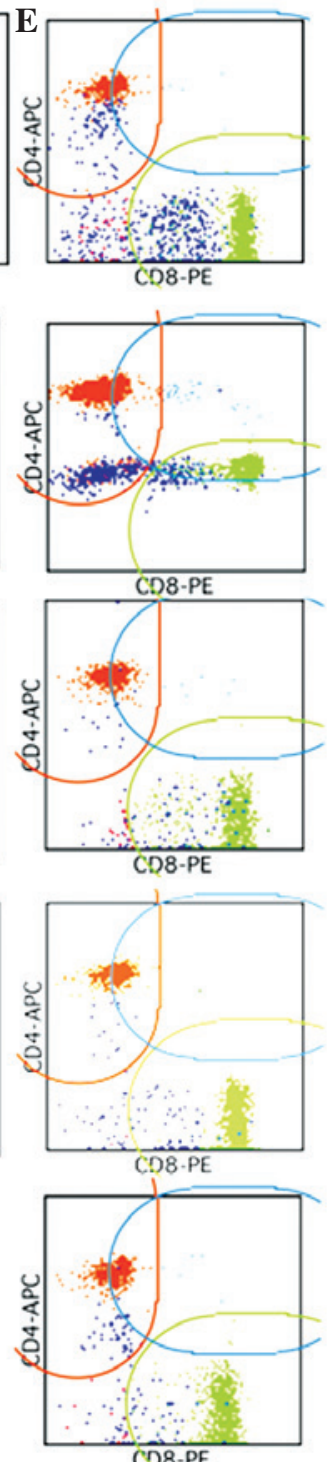

Figure 2. Flow cytometry was used to quantify the patient's peripheral plasma T-lymphocyte subsets as a measure of the patient's immunological status at each time point. The immune status of the patient was changeable; the patient's immunity declined when the tumor was developing and recovered when the tumor was resected. (A) The $\mathrm{CD}^{+} / \mathrm{CD}^{+}$ratios in the primary tumor and metastatic tissues. The $\mathrm{CD}^{+} / \mathrm{CD}^{+}$ratio was $0.37,2.27,0.49,0.49$ and 0.38 in the primary tumor pre-resection, primary tumor post-resection, metastatic colon, metastatic gastric and metastatic lung tissues, respectively. (B) According to the SSC-H set gate to sort granulocytes and monocytes from lymphocytes, the cell in the green circle is a lymphocyte. (C) The lymphocytes were sorted and the cell in the upper and lower left quadrants is a $\mathrm{CD} 3^{-}$cell (B-cell or natural killer cell) and the cell in the upper and lower right quadrants is a $\mathrm{CD} 3^{+}$cell $\left(\mathrm{CD} 4^{+}\right.$or $^{-} \mathrm{CD} 8^{+}$ cell). (D) After sorting the $\mathrm{CD}^{+}$cells, the cell in the red circle is a $\mathrm{CD}^{+}$cell and the cell in the blue circle is a CD3- cell. (E) After sorting for CD3 ${ }^{-}$cells, the cell in the upper left quadrant is a $\mathrm{CD}^{+} \mathrm{CD} 4^{+}$cell (helper/inducer T lymphocyte) and the cell in the lower right quadrant is a $\mathrm{CD} 3^{+} \mathrm{CD} 8^{+}$cell (cytotoxic $\mathrm{T}$ lymphocyte). SSC-H, side scatter height; Hi, high; Lo, low.

occurrence of metastatic lesions in the stomach of $5.4 \%$ of patients examined. Furthermore, lung, breast and esophageal tissues were common primary sites, and malignant melanoma was the most frequent tumor to metastasize to the stomach (29.6\%) (16). Gastric metastases from primary liver cancers are extremely rare. Dormant site metastasis from HCC has been reported in gallbladder, pancreatic tail, oral cavity, skin and soft tissue (3-6); however, this has not yet been reported in the colon or stomach of a single patient following surgical resection. In the present case, the patient responded well to treatment and showed strong indications of health during the follow-up period, in particular following the two surgical resections of the distal metastases. In addition, his immune status improved following resection of the metastatic tumors.
Gastrointestinal metastatic carcinoma displays few specific clinical manifestations, and is thus difficult to distinguish from primary gastric cancer or colorectal cancer (17). The most common manifestations of gastrointestinal metastatic carcinoma include loss of appetite, abdominal pain, vomiting, bleeding, anemia and other digestive tract symptoms (18). Among these symptoms, anemia is typically the primary reason for hospitalization (18). Clinical diagnosis of gastric metastasis is dependent on the medical history of primary tumor and pathological examination (18). Preoperative diagnosis is difficult, and tumor marker inspection may lead to a more accurate diagnosis (18). Concerning therapy for extrahepatic metastasis of liver cancer, Jung et al (19) determined, based on clinical observations, that surgical resection was the most 
A

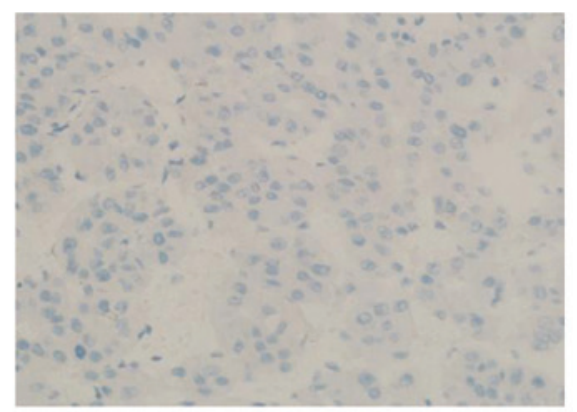

B

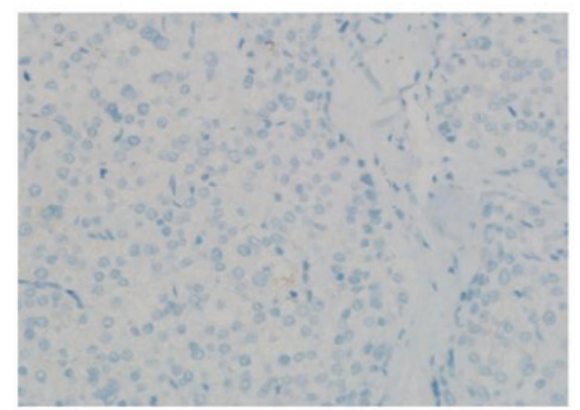

C

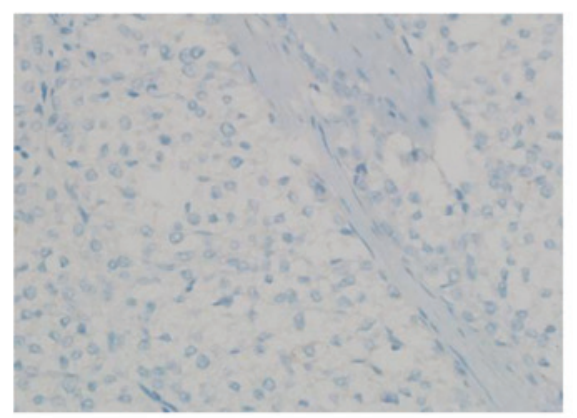

D

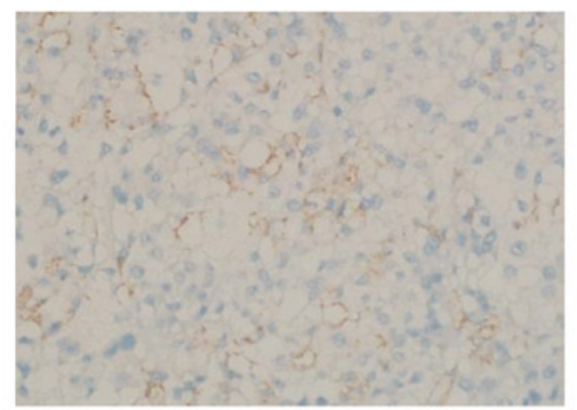

Figure 3. Expression of Fas was detected by immunohistochemistry (magnification, x40). Fas was not in the tumor cells. (A) Fas showed negative expression in (A) primary liver cancer, (B) ascending colon metastatic tumor and (C) gastric metastatic tumor tissue. (D) Fas-positive pathological control section.

useful therapeutic intervention method for metastatic gastric cancer and should be considered as the primary treatment. We followed our patient for 9 years and 2 months, following an initial diagnosis of primary liver cancer until mortality. For the last 3 years and 10 months following resection of the colon metastatic tumor, the patient appeared to be disease-free, suggesting that resection of extrahepatic metastases may result in a good prognosis.

Cancer metastasis is a complex biological progression involving multiple steps and factors (20). Immunosuppression and evasion of immune surveillance by cancer cells may be
A

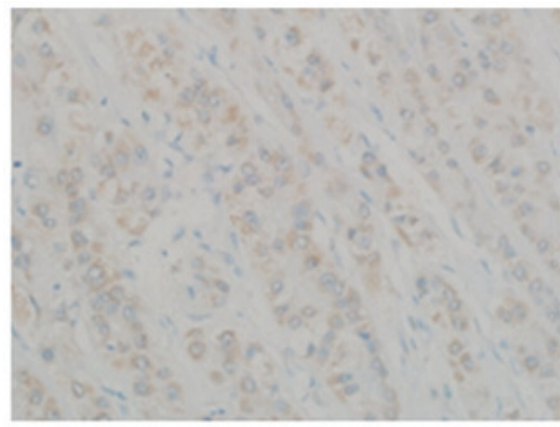

B

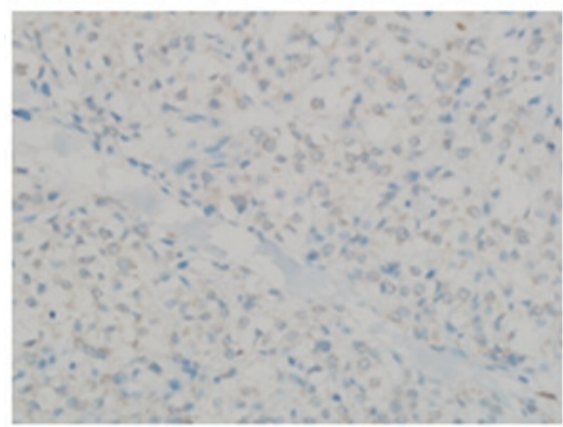

C

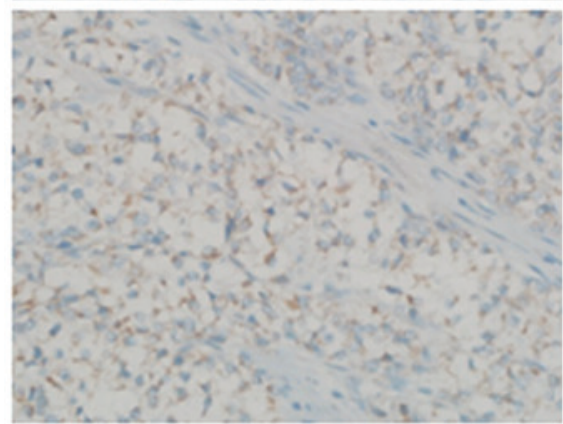

D

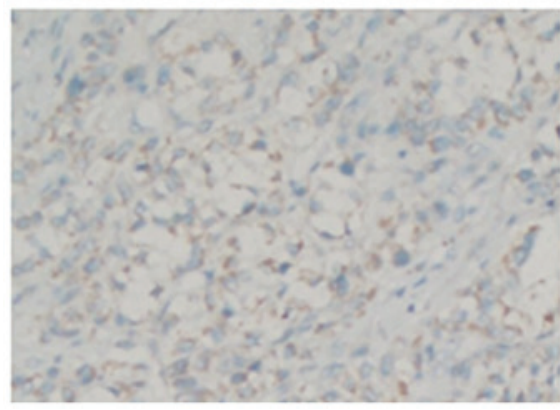

Figure 4. Expression of FasL was detected by immunohistochemistry. FasL was expressed in the cytoplasm of the tumor cells (magnification, $\mathrm{x} 40$ ). FasL showed positive expression in the (A) primary liver cancer, (B) ascending colon metastatic tumor and (C) gastric metastatic tumor tissues. (D) FasL-positive pathological control section.

important mechanisms in this process (20). The role of the immune system in the control of solid tumors has been examined in mouse models (21), but remains poorly understood. Türbachova et al (10) suggested that non-pathological immune tolerance values observed in the peripheral blood of patients with immune-privileged ovarian tumors were sufficient to prevent hematogenous spread following primary diagnosis. By contrast, non-immune-privileged tumors established high immune tolerance in an immunological environment equivalent to the bloodstream, and thus spread hematogenously to distant organs, suggesting that immune tolerance is a powerful marker for tumor aggressiveness and disease 
A

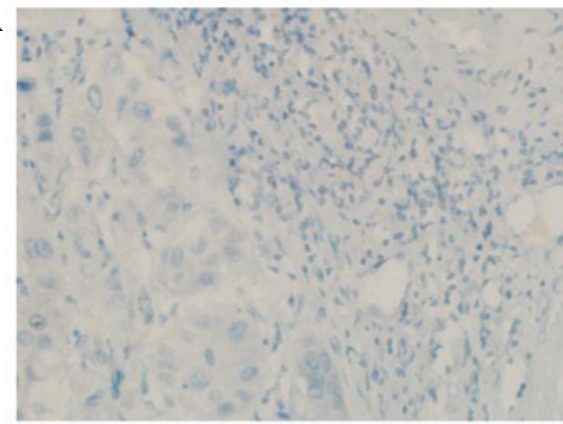

B

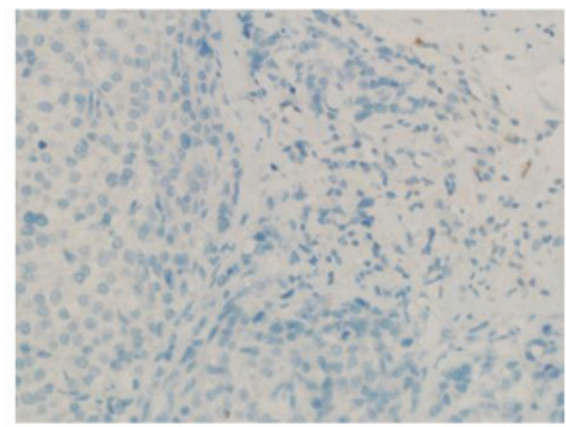

$\mathbf{C}$

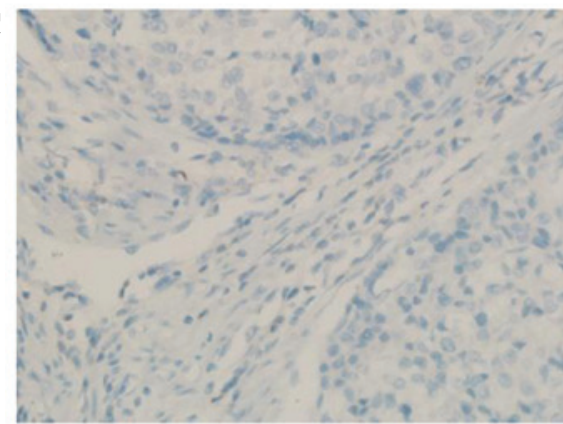

D

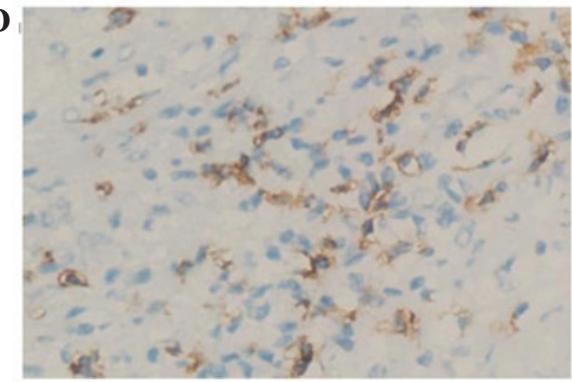

Figure 5. Expression of $\mathrm{CD} 4^{+}$was detected by immunohistochemistry. $\mathrm{CD} 4^{+}$ T-cells were not detected in the stroma of the (A) primary liver cancer, (B) ascending colon metastatic tumor and (C) gastric metastatic tumor tissues (magnification, $\mathrm{x} 40$ ). (D) $\mathrm{CD} 4^{+} \mathrm{T}$-cell-positive pathological control section.

dissemination (10). Camus et al (8) investigated various cellular and molecular parameters in human colorectal cancer, and described an in situ immune response using clinical parameters for metastatic lymph node or distant organ invasion which established an association between intratumoral immune reaction and human colorectal cancer recurrence.

Immune system evasion mechanisms may differ between tumor developmental stages (22). Liver cancer cells may alter the antigenicity of the cell membrane or secrete immune inhibitors to block normal immune function, thus evading immune surveillance and leading to metastasis (23). The Fas/FasL-related immune evasion mechanism is closely
$\mathbf{A}$

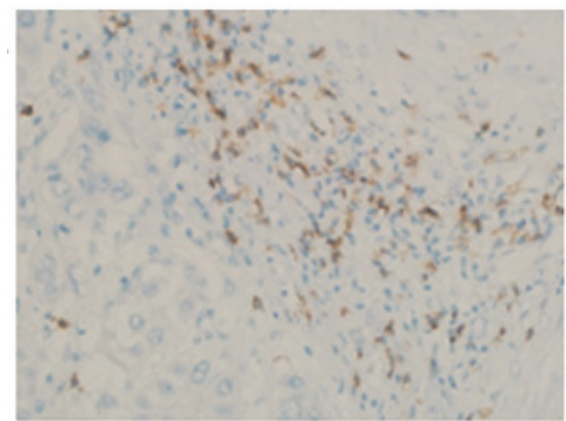

B

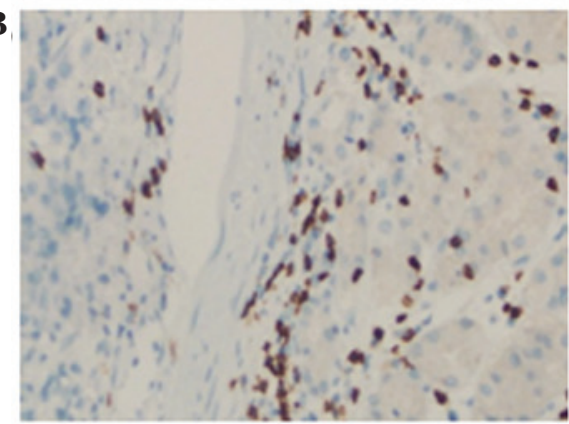

C
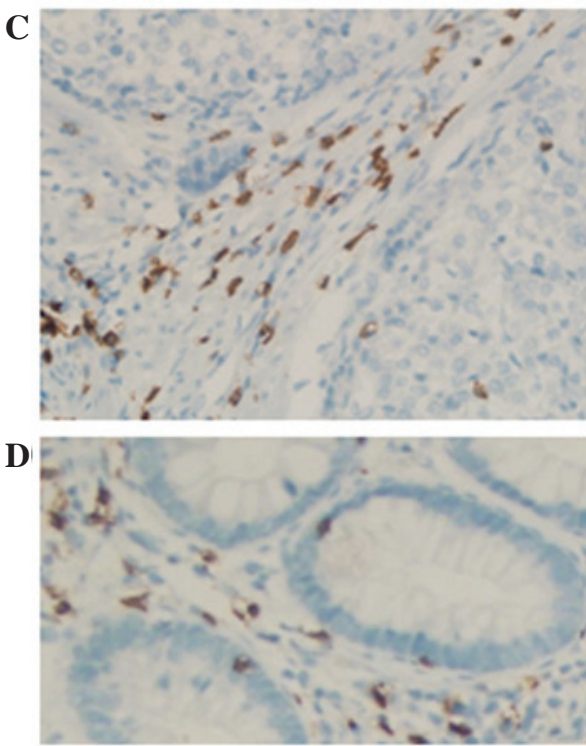

Figure 6. Expression of $\mathrm{CD}^{+}$was detected by immunohistochemistry. $\mathrm{CD}^{+} \mathrm{T}$-cells were detected in the stroma of the (A) primary liver cancer, (B) ascending colon metastatic tumor and (C) gastric metastatic tumor tissues (magnification, $\mathrm{x} 40$ ). (D) $\mathrm{CD}^{+} \mathrm{T}$-cell-positive pathological control section.

associated with metastasis (24). Fas is able to induce the apoptosis of various cell types via interaction with FasL and the TNF receptor superfamily member 6 death receptor (Fas receptor), and has been shown to induce apoptosis of immature thymocytes during thymic selection (25). He et al (26) reported that the upregulation of FasL expression and reduction in tumor-infiltrating lymphocytes apoptosis in Epstein-Barr virus-associated gastric carcinoma may facilitate the evasion of tumor cells from the host immunosurveillance, and may contribute to the development and progression of carcinoma. In the present patient, higher FasL expression was detected in liver cancer cells, while lower expression of Fas was observed. These results suggested that the change in Fas/FasL expression stimulated apoptosis of immune cells and reduced the patient's immune function. As a result, HCC spread to the colon and 
stomach 5 years after resection of the primary HCC, during a time when the immune system activity was depressed. The development and spread of metastatic tumors may thus be associated with changes in immune function.

The present case report aimed to investigate whether the Fas/FasL system mediates immune system suppression and allow tumors to evade immune surveillance. In vitro experiments have shown that Fas is expressed in the majority of cancer cells and that FasL-expressing cancer cells mediate Fas-induced T-cell destruction, which may protect the organ against attack by the immune system (27). Tumors may thus be regarded as immune privileged areas. In addition, numerous types of tumor cell are resistant to Fas-mediated apoptosis, for unknown reasons (28). Results from monitoring the present patient's immune status throughout his disease progression showed that during active metastasis, $\mathrm{CD}^{+}$and $\mathrm{CD} 8^{+} \mathrm{T}$ cell populations in the blood were reduced, and the $\mathrm{CD} 4^{+} / \mathrm{CD}^{+}$ ratio was reduced. Using flow cytometric analysis to quantify the patient's peripheral plasma T-lymphocyte subsets, the $\mathrm{CD}^{+} / \mathrm{CD}^{+}$ratios were $0.37,0.49,0.49$ and 0.38 in the liver cancer, metastatic colon, metastatic gastric and metastatic lung tumor tissues, respectively. This pattern was reversed when the patient was free from tumor, the quantitative value recovered to 2.27 when the primary liver tumor had been resected. However, FasL showed positive expression in tumor tissues while Fas was negative, indicating that tumor development may be coupled to changes in immune status. It is possible that tumor resection allowed for the recovery of the patient's immune status, which modulated growth of remnant tumor cells in the body. When the immune system was suppressed, the remnant tumor cells may have proliferated, leading to recurrence or metastasis, and mortality.

This is an atypical case, with primary HCC metastasized sequentially to colon, stomach and lung. The present report highlights the importance of closely monitoring patients for possible occurrence of metastatic tumors at rare sites. Furthermore, although alterations in immune status have previously been noted following tumor metastasis (10), the present report delineates the types of changes that may occur in the immune system during disease progression. During our patient's $>9$-year survival period, available tissues facilitated the investigation of the association between immune changes and disease failure. Further clinical research will be required to validate the present hypothesis regarding the involvement of the immune system in cancer metastasis.

\section{Acknowledgements}

This study was supported by the natural science foundation of Hubei province, China (grant no. 2013CFA078). The words of the manuscript were revised by International Science Editing.

\section{References}

1. Verdecchia A, Francisci S, Brenner H, Gatta G, Micheli A, Mangone L and Kunkler I; EUROCARE-4 Working Group Recent cancer survival in Europe: A 2000-02 period analysis of EUROCARE-4 data. Lancet Oncol 8: 784-796, 2007.

2. Natsuizaka M, Omura T, Akaike T, Kuwata Y, Yamazaki K, Sato T, Karino Y, Toyota J, Suga T and Asaka M: Clinical features of hepatocellular carcinoma with extrahepatic metastases. J Gastroenterol Hepatol 20: 1781-1787, 2005.
3. Ueno N, Kanamaru T, Kawaguchi K, Tanaka K, Inoue K, Idei Y and Yamamoto M: A hepatocellular carcinoma with lymph node metastasis and invasion into the gallbladder: Preoperative difficulty ruling out a gallbladder carcinoma. Oncol Rep 8: 331-335, 2001.

4. Texler ML, Pierides J and Maddern GJ: Case report: A hepatocellular carcinoma metastasis in the distal pancreas. J Gastroenterol Hepatol 13: 467-470, 1998.

5. Hyun YS, Choi HS, Bae JH, Jun DW, Lee HL, Lee OY, Yoon BC, Lee MH, Lee DH, Kee CS, et al: Chest wall metastasis from unknown primary site of hepatocellular carcinoma. World J Gastroenterol 12: 2139-2142, 2006.

6. Tearda T and Sugiura M: Metastatic hepatocellular carcinoma of the skin diagnosed with hepatocyte paraffin 1 and $\alpha$-fetoprotein immunostainings. Int J Surg Pathol 18: 433-436, 2010.

7. Terada $\mathrm{T}$ and Maruo H: Unusual extrahepatic metastatic sites from hepatocellular carcinoma. Int J Clin Exp Pathol 6: 816-820, 2013.

8. Camus M, Tosolini M, Mlecnik B, Pagès F, Kirilovsky A, Berger A, Costes A, Bindea G, Charoentong P, Bruneval P, et al: Coordination of intratumoral immune reaction and human colorectal cancer recurrence. Cancer Res 69: 2685-2693, 2009.

9. Möller P1, von Reyher U, Leithäuser F and Sträter J: CD95 (APO-1/Fas) and CD95-ligand (CD95L). Implications of these apoptosis mediating receptor/ligand systems in the pathogenesis of autoimmune diseases. Verh Dtsch Ges Pathol 80: 12-20, 1996 (In German).

10. Türbachova I, Schwachula T, Vasconcelos I, Mustea A, Baldinger $\mathrm{T}$, Jones $\mathrm{KA}$, Bujard $\mathrm{H}$, Olek A, Olek $\mathrm{K}$, Gellhaus K, et al: The cellular ratio of immune tolerance (immunoCRIT) is a definite marker for aggressiveness of solid tumors and may explain tumor dissemination patterns. Epigenetics 8: 1226-1235, 2013.

11. Rutherford AE, Hynan LS, Borges CB, Forcione DG, Blackard JT, Lin W, Gorman AR, Shaikh OS, Reuben A, Harrison E, et al: Serum apoptosis markers in acute liver failure: A pilot study. Clin Gastroenterol Hepatol 5: 1477-1483, 2007.

12. Bosman FT (ed); World Health Organization (WHO); International Agency for Research on Cancer (IARC): WHO classification of tumours of the digestive system. 4th edition. IARC Press, Lyon, France, pp212-216, 2010.

13. Kolokythas A, Weiskopf S, Singh M and Cabay RJ: Renal Cell Carcinoma: Delayed Metachronous Metastases to Parotid and Cerebellum. J Oral Maxillofac Surg 73: 1296-1303, 2015.

14. Campoli PM,Ejima FH, Cardoso DM, Silva OQ, Santana Filho JB, Queiroz Barreto PA, Machado MM, Mota ED, Araujo Filho JA, Alencar Rde C and Mota OM: Metastatic cancer to the stomach. Gastric Cancer 9: 19-25, 2006.

15. Amicucci G, Sozio ML, Sozio A and Rizzo FM: Gastric metastases of breast carcinoma. Am J Gastroenterol 94: 859, 1999.

16. Oda, Kondo H, Yamao T, Saito D, Ono H, Gotoda T, Yamaguchi H, Yoshida S and Shimoda T: Metastatic tumors to the stomach: Analysis of 54 patients diagnosed at endoscopy and 347 autopsy cases. Endoscopy 33: 507-510, 2001

17. Gopalan S, Bose JC and Periasamy S: Anastomotic Recurrence of Colon Cancer-is it a Local Recurrence, a Second Primary, or a Metastatic Disease (Local Manifestation of Systemic Disease)? Indian J Surg 77: 232-236, 2015.

18. Menuck LS and Amberg JR: Metastatic disease involving the stomach. Am J Dig Dis 20: 903-913, 1975.

19. Jung HJ, Lee HY, Kim BW, Jung SM, Kim HG, Ji JS, Choi H and Lee BI: Gastic metastasis from ovarian adenocarcinoma presenting as a submucosal tumor without ulceration. Gut Liver 3: 211-214, 2009.

20. Mellman I, Coukos G and Dranoff G: Cancer immunotherapy comes of age. Nature 480: 480-489, 2011.

21. Kaplan DH, Shankaran V, Dighe AS, Stockert E, Aguet M, Old LJ and Schreiber RD: Demonstration of an interferon gamma-dependent tumor surveillance system in immunocompetent mice. Proc Natl Acad Sci USA 95: 7556-7561, 1998.

22. Campoli M, Ferrone S, Zea AH, Rodriguez PC and Ochoa AC: Mechanisms of tumor evasion. Cancer Treat Res 123: 61-88, 2005.

23. Sun AM, Li CG, Zhang YQ, Lin SM, Niu HR and Shi YS: Hepatocarcinoma cell-derived hepatoma-derived growth factor (HDGF) induces regulatory T cells. Cytokine 72: 31-35, 2015.

24. Gordon $\mathrm{N}$ and Kleinerman ES: The role of Fas/FasL in the metastatic potential of osteosarcoma and targeting this pathway for the treatment of osteosarcoma lung metastases. Cancer Treat Res 152: 497-508, 2009. 
25. Pezzano M, Samms M and Guyden JC: TNF and Fas-induced apoptosis during negative selection in thymic nurse cells. Ethn Dis 11: 154-156, 2001.

26. He D, Xiao L, Chen JN, Liang Q and Shao CK: Correlation of Fas/FasL expression to cell apoptosis in Epstein-Barr virus-associated gastric carcinoma. Chin J Cancer 29: 283-287, 2010.
27. O'Connell J, O'Sullivan GC, Collins JK and Shanahan F: The Fas counterattack: Fas-mediated T cell killing by colon cancer cells expressing Fas ligand. J Exp Med 184: 1075-1082, 1996.

28. Strand $S$ and Galle PR: Immune evasion by tumours: Involvement of the CD95 (APO-1/Fas) system and its clinical implications. Mol Med Today 4: 63-68, 1998. 